\title{
Measurement of the re-entrant lepton spectrum with the High-Energy Particle Detector on board CSES-01
}

\section{Alessandro Sotgiu ${ }^{a, b, *}$ on behalf of the CSES-Limadou Collaboration}

(a complete list of authors can be found at the end of the proceedings)

"University of Rome "Tor Vergata” (Department of Physics),

via della Ricerca Scientifica 1, Rome, Italy

bINFN-Section of Rome "Tor Vergata",

via della Ricerca Scientifica 1, Rome, Italy

E-mail: alessandro.sotgiu@roma2.infn.it

The High-Energy Particle Detector (HEPD-01) is one of the particle detectors installed on board the China Seismo-Electromagnetic Satellite (CSES-01). The instrument consists of different subdetectors, including two planes of double-sided silicon microstrip sensors, a calorimeter constituted by 16 plastic scintillators and a layer of LYSO crystals, and a scintillator veto system surrounding the calorimeter. The detector is dedicated to the measurement of proton (30-200 $\mathrm{MeV}$ ) and electron (3-100 MeV) fluxes, and their variations induced by short-time perturbations of the radiation belts due to solar, terrestrial, or anthropic phenomena. Although the detector is capable to measure particles with a galactic origin, due to its energy range and to the CSES-01 polar orbit, HEPD collects particles below the local geomagnetic cutoff for a large fraction of its total live time. In this work, the differential spectrum of re-entrant leptons (the downward-moving component of secondary electrons and positrons produced in the interactions of cosmic ray protons with the atmosphere) is measured in the near-equatorial region (altitude about $500 \mathrm{~km}$ ) in the energy interval between 10 and $100 \mathrm{MeV}$ where there is a lack of recent experimental data.

$37^{\text {th }}$ International Cosmic Ray Conference (ICRC 2021)

July 12th - 23rd, 2021

Online - Berlin, Germany

\footnotetext{
*Presenter
} 


\section{Introduction}

High-energy galactic particles (mainly protons and helium nuclei) can penetrate the Earth's magnetic field and interact in atmosphere leading to the production of charged secondary particles. Some of these secondaries move in the upward direction along the field line (the so-called splash albedo) and, if their rigidity is higher than the geomagnetic cut-off, they can escape to the interplanetary space. Otherwise, if these particles have a rigidity below the geomagnetic cut-off, they spiral along the field line and re-enter in the opposite hemisphere with a downward direction (re-entrant albedo).

Albedo particles are studied since the 50s with a lot of balloons experiment during the 2nd half of the last century[1], [2]. However, such kinds of experiments presented some limitations, as for example the large background due to atmospheric particles or the limited regions of space that could be observed with a balloon-borne experiment. With spacecraft experiments, it became possible to measure the differential energy spectrum of albedo particles in various regions of the near-Earth space. Electrons and positrons, produced by pion decays, and protons are the dominating and the most studied component of albedo particles, but some contradictions between experimental data and theoretical predictions are still present[3].

CSES-01 (China Seismo-Electromagnetic Satellite)[4] is the first of a series of scientific space missions dedicated to monitoring electromagnetic fields and waves, plasma and particle perturbations of the atmosphere, ionosphere, and magnetosphere. Currently, the CSES-01 satellite is in orbit since February 2nd, 2018 equipped with nine instruments, among them the Italian High-Energy Particle Detector (HEPD-01). Data collected during the missions also allow studying solar-terrestrial interactions and solar physics phenomena, namely Coronal Mass Ejections (CMEs), solar flares, and cosmic ray solar modulation. For most of its acquisition time along CSES-01 orbits, HEPD-01 collects particles with energy below the local geomagnetic cutoff, thus being perfectly suited for the study of the albedo component. In this work, the method of re-entrant leptons identification and a preliminary result of the measured spectrum is reported.

\section{HEPD-01}

The High-Energy Particle Detector[5] (HEPD-01) is one of the payloads installed onboard the CSES-01 satellite. It is devoted to the measurements of electrons (3-100 MeV) and protons (30-200 MeV) fluxes and their variations. The detector includes the following set of sub-detectors (in descending order from the top to the bottom side of the instrument):

- the tracker plane - made of two layers of double-sided silicon strip sensors - for the reconstruction of the incoming particle direction

- the trigger plane - made of a segmented layer of 6 plastic scintillator bars - for the generation of the trigger pulse

- the calorimeter - made of a plastic scintillator tower (16 planes) plus a final layer of LYSO inorganic crystals - for the measurement of the energy deposition 


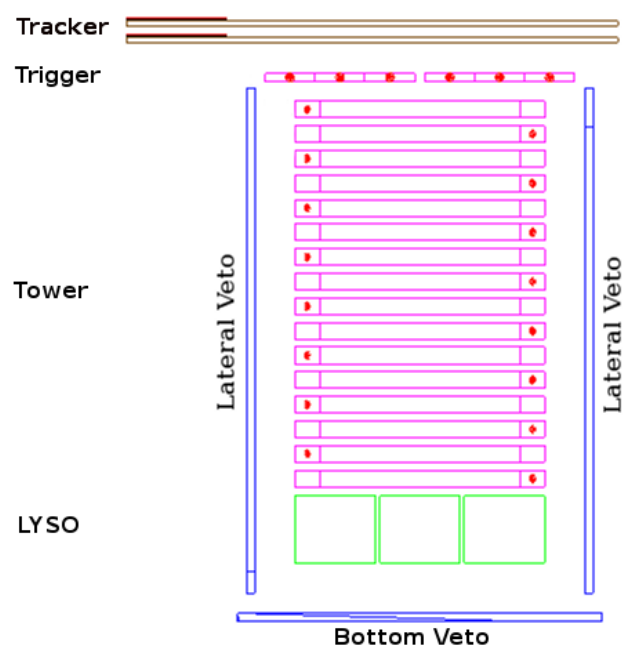

(a)

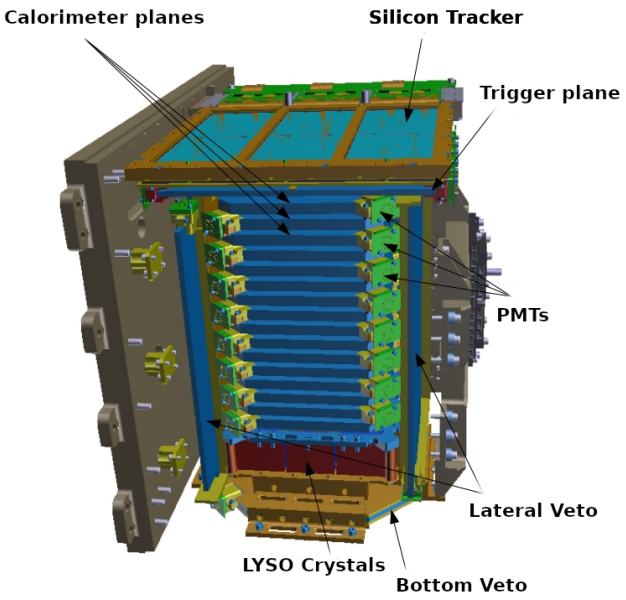

(b)

Figure 1: (a) A scheme of the HEPD-01 detector, as implemented in the Geant 4 software used for the detector simulations. The red dots correspond to the positions where the Photo-Multiplier Tubes are placed. (b) Computer-aided design of the HEPD-01 detector, including the mechanical structures and the satellite wall where the detector is anchored. In both pictures, the frontal and posterior veto panels have been removed for visualization purposes.

- a veto system - made of 5 plastic scintillator planes - that surround the calorimeter (four later planes plus a bottom one) for the rejection of particles not fully contained inside the calorimeter

The trigger plane, as well as the calorimeter and the veto system, are read-out by PhotoMultipliers Tubes. A scheme of the detector is reported in Fig. 1 with (a) showing only the sub-detectors as implemented in the Monte Carlo software and (b) showing the complete apparatus including the mechanical structures. A detailed description of the detector can be found in [6] and [7].

The detector is acquiring data since the CSES-01 launch (February 2018), in the latitude region between $\simeq-65^{\circ}$ and $\simeq+65^{\circ}$. During the polar passages, it is switched off because of satellite attitude operations.

\section{Data sample and analysis}

During the HEPD-01 in-flight acquisition runs, an event is recorded when a particle deposits a signal above the configured threshold in both the segmented trigger plane (T) and the first two planes of the plastic scintillator tower (P1 and P2). Further selections are then applied offline, during the data analysis, in order to clean the sample as much as possible. These selections include: 
- the containment. The signals on the five anti-coincidence planes (four lateral and one bottom) are requested to be below the thresholds. This condition ensures a proper reconstruction of the deposited energy of the event;

- for each event, only one of the six trigger bars can be hit (i.e. trigger multiplicity equals 1 ). This is used to reject multi-particle events or events with the production of secondaries inside the calorimeter;

- a continuous release of signal inside the scintillator tower. This condition requires that, if the signal on the $i$-th plane is below the threshold, then the signal of all the deeper planes from $P i$ to the last one P16, and also the signal on the final LYSO layer, must be below the thresholds. This is used to reject interacting events that can produce neutral secondaries (for example because of inelastic interactions) that can cross a part of the calorimeter without releasing any signal and then interact again at the bottom part of the calo.

The geometrical factor of HEPD-01 is evaluated using a Monte Carlo (MC) simulation (Geant4based software) of isotropically generated electrons, from $1 \mathrm{MeV}$ to $200 \mathrm{MeV}$, after applying the trigger conditions used in-flight (i.e. signal on $T \& P 1 \& P 2$ ) and the containment request (i.e. no hit on the anti-coincidence planes). Our MC software includes the generation and propagation of the scintillation light produced in the calorimeter by the passage of the charged particles and its collection on the PMTs. This process takes into account the efficiency of the scintillator material and the quantum efficiency of the PMTs. The number of photo-electrons collected in each PMT is then converted in an ADC signal after a proper tuning with HEPD-01 test beam data.

The same MC software is also used for the evaluation of all the selection cuts efficiencies.

In order to discriminate electrons and positrons from protons (the most important source of background), ionization energy losses inside each calorimeter plane are required to be compatible with the expectation for a singly charged minimum ionizing particle (mip). This selection takes advantage of the modular structure of the scintillator tower and the different range of protons and electrons in the HEPD-01 energy range for fully contained particles. In particular, for each plane $i$ in which the particle can be stopped, a maximum allowed signal is defined for all the $P j$ with $j<i$. These maximum allowed signals for each plane have been defined by means of MC simulations, as well as the resulting efficiency of this selection cut.

In addition to the previous set of selection criteria, used to obtain a clean sample of leptons, specific selections are then applied for the analysis of the re-entrant albedo component. At first, particles have been selected in the L-shell geomagnetic region between 1.1 and 1.2. The L-shell, or McIlwain L-parameter, is a parameter describing a particular set of planetary magnetic field lines. Inside HEPD-01 data, it is calculated with a temporal precision of 1 second, considering the CSES01 satellite position and using the IGRF-13 model for the approximation of the Earth's magnetic field (B). In Fig. 2, the region in the latitude-longitude map corresponding to the L-shell cut is reported for CSES-01 orbits. The colored scale reports the value of the energy cutoff, expressed in $\mathrm{MeV}$, at the altitude of CSES-01. As can be noted, an energy higher than $8 \mathrm{GeV}$ is needed for a galactic electron to reach the detector position, while the request of containment inside the HEPD-01 calorimeter limits the maximum lepton energy to $\simeq 100 \mathrm{MeV}$. Therefore, all the contained leptons selected in this region are secondaries produced in the atmosphere. The region corresponding to 


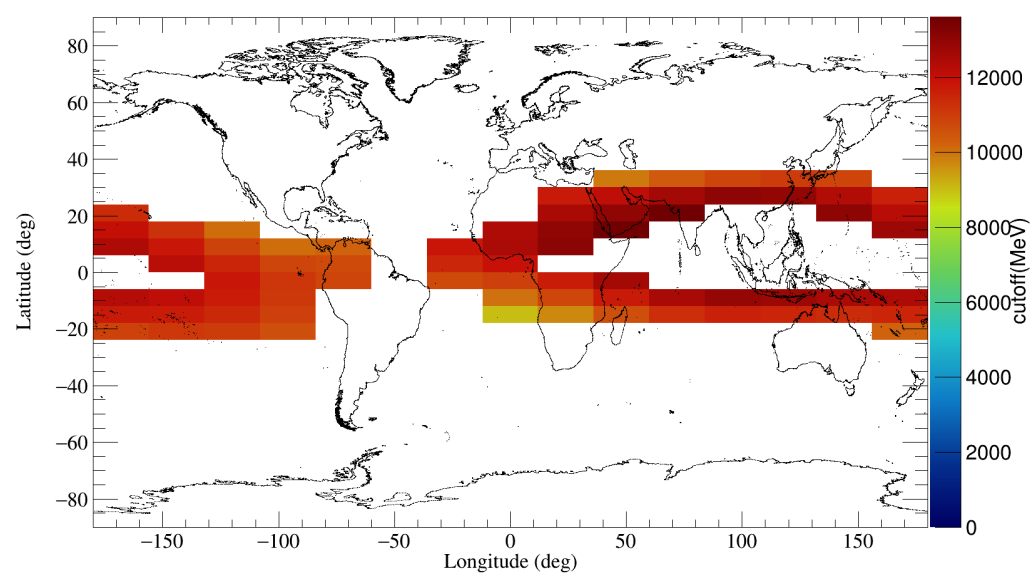

Figure 2: The region corresponding to the selections $1.1<\mathrm{L}$-shell $<1.2$ and B > 23000 nT. The colored scale represents the value of the energy cutoff expressed in MeV. Considering the HEPD-01 electron energy range for contained particles $(3-100 \mathrm{MeV})$ it is clear that in this region the observed lepton cannot have a galactic origin.

the South Atlantic Anomaly (SAA), where mostly trapped particles can be detected, is rejected by selecting $\mathrm{B}>23000 \mathrm{nT}$.

The request of no signal on the bottom veto plane is also sufficient to discard the splash albedo component with an upward direction.

The detector's live time is calculated after every event and corresponds to the time from the end of the processing of the previous event and the arrival of the current event. It is the time during which the detector is capable to acquire a new event and its measurement is performed by the trigger electronics board. For this analysis, HEPD-01 is considered always sensitive to re-entrant albedo particles, thus the live time is accumulated considering all the time spent by the apparatus in the orbital position selected by the aforementioned selection criteria $(1.1<\mathrm{L}<1.2$ and $\mathrm{B}>23.000 \mathrm{nT})$.

Residual background due to misidentified interacting protons with energy $>500 \mathrm{MeV}$ has been evaluated with a proton MC simulation reproducing the galactic proton spectrum in the same L-region. This background, mostly due to the inelastic production of leading neutral barions, is less than $5 \%$ on the whole energy range and has been included in the systematic uncertainty of the final spectrum.

\section{Results and Conclusions}

HEPD-01 data, recorded during August2018 (immediately after the end of the detector commissioning), were used to determine the preliminary re-entrant all-electrons flux reported in Fig. 3. The resulting spectrum is shown together with PAMELA measurements from [8] (acquired in the same L-shell region), after the sum of the electron and positron components and with a theoretical model[9] used for the calculation of secondary electron and positron fluxes at the top of atmosphere with energy greater than $10 \mathrm{MeV}$. The error bars on the HEPD-01 points include the 


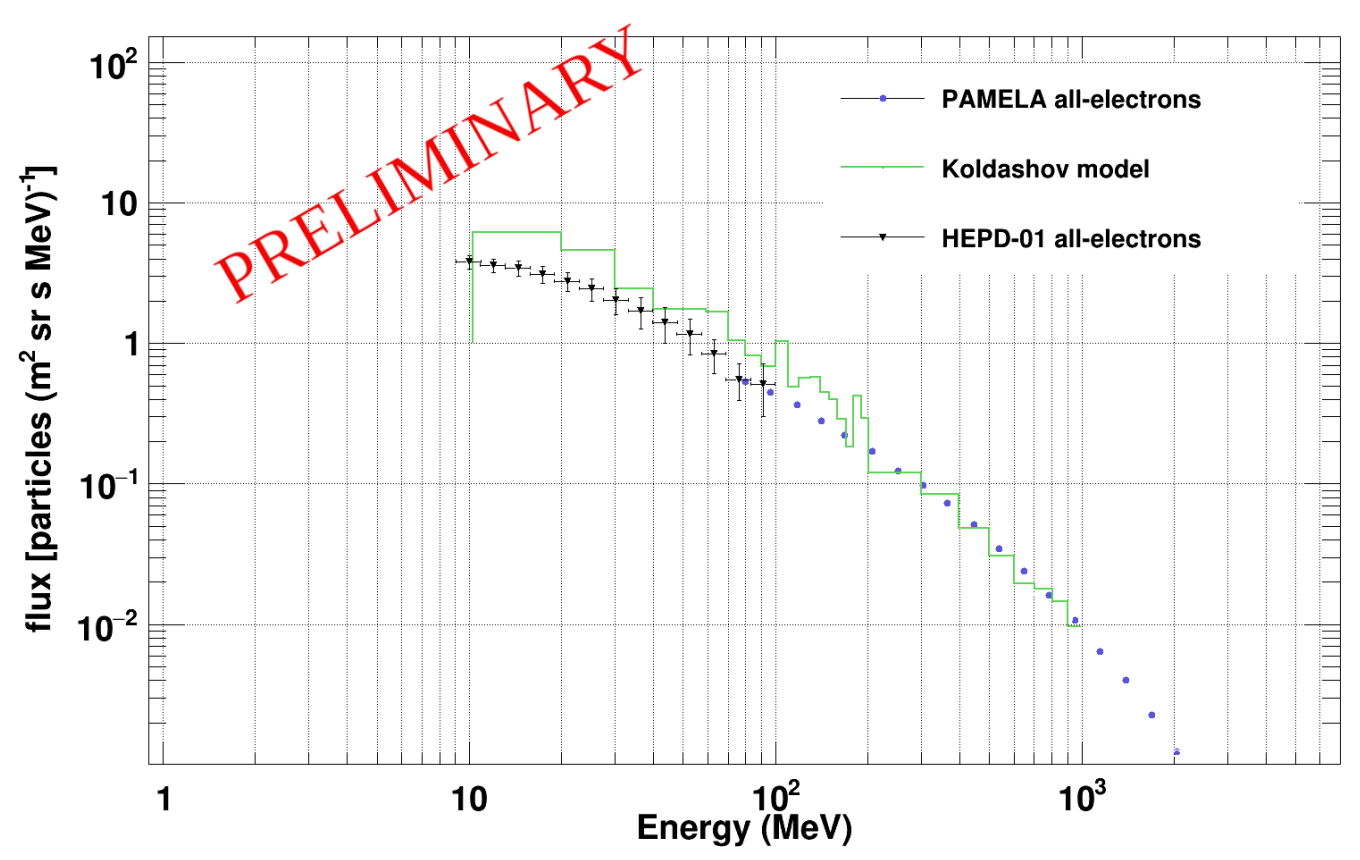

Figure 3: Re-entrant all-electrons differential flux measured by HEPD-01 (black points) and compared with PAMELA data[8] (blue points) and theoretical model[9] (the green line).

statistical error, which is small due to large detector acceptance and the high collected statistics, and systematic uncertainties. The latter includes the differences between MC simulation and flight data that are still present after the digitization, the limited statistics of the simulated samples used for the calculation of the selection cut efficiencies and the geometrical factor, and the high-energy protons contamination.

HEPD-01 and PAMELA are in good agreement in the region where the instruments' energy ranges overlap, while the model predicts higher fluxes at low energy with respect to the observed ones. To finalize the HEPD-01 measurement further studies are required to extend the analyzed time period, to inspect other L-shell regions, and to include the measurement below $10 \mathrm{MeV}$. The HEPD-01 will provide important data about the re-entrant electron component below $100 \mathrm{MeV}$ where there is a substantial lack of recent data and will help the development of secondary particle production models.

\section{References}

[1] Verma S.D. et al., Measurement of Charged Splash and Re-entrant Albedo of the Cosmic Radiation. , Journal of Geophysical Researches, 72 (1967)

[2] Wenzel K.P. et al., Splash Albedo Protons Between 4 and $315 \mathrm{MeV}$ at High and Low Geomagnetic Latitude. , Journal of Geophysical Researches, 80:3580-3584 (1975) 
[3] Mayorov, A. et al., Splash and Re-entrant Albedo Fluxes Measured in the PAMELA Experiment., Physics Procedia. Volume 74, Pages 314-319, (2015)

[4] X. Shen, et al., The state-of-art of the China Seismo-Electromagnetic Satellite mission, Science China Technological Sciencies, vol. 61, no. 5, pp.634-642, May 2018.

[5] G. Ambrosi, et al., The HEPD particle detector of the CSES satellite mission for investigating seismo-associated perturbations of the Van Allen belts, Sci China Tech Sci, 2018, 61: 643-652.

[6] G. Ambrosi, et al., Beam test calibrations of the HEPD detector on board the China SeismoElectromagnetic Satellite, Nuclear Inst. and Methods in Physics Research, A 974 (2020) 164170, September 2020.

[7] P. Picozza, et al., Scientific Goals and In-orbit Performance of the High-energy Particle Detector on Board the CSES, The Astrophysical Journal Supplement Series, vol. 243, pp. 16, July 2019.

[8] O. Adriani, et al., Measurements of quasi-trapped electron and positron fluxes with PAMELA, in Journal of Geophysical Research, 114 (2009)

[9] Koldashov, S. V. ; Mikhailov, V. V. ; Voronov, S. A. Electron and Positron Albedo Spectra with Energy more than $20 \mathrm{MeV}$, in International Cosmic Ray Conference 24th, Rome, vol. 4 , pp. 993 - 996, Int. Union of Pure and Appl. Phys., Rome. 


\section{Full Authors List: CSES-Limadou Collaboration}

S. Bartocci ${ }^{1}$, R. Battiston ${ }^{2,3}$, F. Benotto ${ }^{4}$, S. Beole ${ }^{4,5}$, W.J. Burger ${ }^{3,6}$, D. Campana ${ }^{7}$, G. Castellini ${ }^{8}$, P. Cipollone ${ }^{1}$, S. Coli ${ }^{4}$, L. Conti ${ }^{1,9}$, A. Contin ${ }^{10,11}$, M. Cristoforetti ${ }^{12}$, L. De Cilladi ${ }^{4,5}$, C. De Donato ${ }^{1}$, C. De Santis ${ }^{1}$, F.M. Follega ${ }^{2,3}$, G. Gebbia ${ }^{2,3}$, R. Iuppa ${ }^{2,3}$, M. Lolli $^{11}$, N. Marcelli $i^{1,13}$, M. Martucci ${ }^{1,13}$, G. Masciantonio ${ }^{1}$, M. Mergé ${ }^{1, \dagger}$, M. Mese ${ }^{7,14}$, C. Neubuser ${ }^{3}$, F. Nozzoli ${ }^{3}$, A. Oliva ${ }^{11}$, G. Osteria $^{7}$, L. Pacini ${ }^{15}$, F. Palma ${ }^{1, \dagger}$, F. Palmonari ${ }^{10,11}$, A. Parmentier ${ }^{1}$, F. Perfetto ${ }^{7}$, P. Picozza ${ }^{1,13}$, M. Piersanti ${ }^{16}$, M. Pozzato ${ }^{11}$, E.

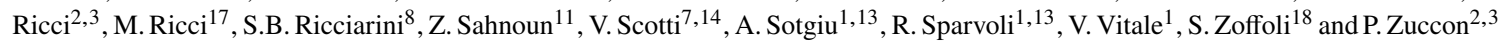

${ }^{1}$ INFN-Sezione di Roma "Tor Vergata”, V. della Ricerca Scientifica 1, I-00133 Rome, Italy;

${ }^{2}$ University of Trento, V. Sommarive 14, I-38123 Povo (Trento), Italy;

${ }^{3}$ INFN-TIFPA, V. Sommarive 14, I-38123 Povo (Trento), Italy;

${ }^{4}$ INFN-Sezione di Torino, Via P. Giuria 1, I-10125 Torino, Italy;

${ }^{5}$ University of Torino, Via P. Giuria 1, I-10125 Torino, Italy;

${ }^{6}$ Centro Fermi, V. Panisperna 89a, I-00184 Rome, Italy;

${ }^{7}$ INFN-Sezione di Napoli, V. Cintia, I-80126 Naples, Italy;

${ }^{8}$ IFAC-CNR, V. Madonna del Piano 10, I-50019 Sesto Fiorentino (Florence), Italy;

${ }^{9}$ Uninettuno University, C.so V. Emanuele II 39, I-00186 Rome, Italy;

${ }^{10}$ University of Bologna, V.le C. Berti Pichat 6/2, I-40127 Bologna, Italy;

${ }^{11}$ INFN-Sezione di Bologna, V.le C. Berti Pichat 6/2, I-40127 Bologna, Italy;

${ }^{12}$ Fondazione Bruno Kessler, V. Sommarive 18, I-38123 Povo (Trento), Italy;

${ }^{13}$ University of Rome "Tor Vergata", V. della Ricerca Scientifica 1, I-00133 Rome, Italy;

${ }^{14}$ University of Naples "Federico II", V. Cintia 21, I-80126 Naples, Italy;

15 INFN-Sezione di Firenze, V. Sansone 1, I-50019 Sesto Fiorentino (Florence), Italy;

${ }^{16}$ INAF-IAPS, V. Fosso del Cavaliere 100, I-00133 Rome, Italy;

${ }^{17}$ INFN-LNF, V. E. Fermi 54, I-00044 Frascati (Rome), Italy;

${ }^{18}$ Italian Space Agency, V. del Politecnico, I-00133 Rome, Italy;

$\dagger$ At ASI Space Science Data Center (SSDC) also, V. del Politecnico, I-00133 Rome, Italy. 